\title{
Bestimmung von Kephalin, Lecithin, Lysolecithin und Sphingomyelin im Serum oder in anderen biologischen Flüssigkeiten
}

\author{
Von P. ZAHLer \\ Aus dem Theodor-Kocher-Institut der Universität Bern (Wissenschaftliche Leitung: Prof. E. F. Lüscher) und dem \\ Zentrallaboratorium des Blutspendedienstes des Schweizerischen Roten Kreuzes (Direktor: Prof. A. Hässig)
}

(Eingegangen am 2. Dezember 1966)

Eine einfache Routine-Methode zur Bestimmung der 4 wichtigsten Scrumphosphatide Lecithin, Lysolecithin, Kephalin und Sphingomyelin wird beschrieben. Die im Detail ausgeführte Vorschrift umfaßt die Lipoidextraktion aus dem Serum, die dünnschichtchromatographische Auftrennung, die Elution der Lipoidfraktionen aus dem Kieselgel, sowie die Phosphorbestimmung. Die Methode erlaubt folgende quantitative Angaben: Gesamtlipoide, Anteil Phosphatide, Anteil Lecithin, Lysolecithin, Kephalin und Sphingomyelin.

A simple routine method is described for determining the four main serum-phosphatides: Lecithin, lyso-lecithin, cephalin and sphingomyelin. The detailed specification covers the extraction of the lipids from the serum, the thin layer chromatographic separation, the elution of the lipid fractions from the silica gel and the determination of phosphorus. This method gives quantitative data on the following: Total lipids and the amounts of phosphatides, lecithin, lysolecithin, cephalin and sphingomyelin.

Durch die Eirführung der Kieselsäure für die Chromatographie der Lipoide durch Kaufmann (1) im Jahre 1939 hat die gesamte Lipoidchemie einen bedeutenden Impuls erhalten, der sich allerdings erst in den Fünfzigerjahren auszuwirken begann. WREN (2) hat 1960 die Erfahrungen mit Kieselsäure in der Säulenchromatographie beschrieben und MARINETTI (3) hat die Resultate der in den USA verwendeten Chromatographie auf kieselsäureimprägniertem Papier zusammengefaßt. Die ersten dünnschichtchromatographischen Trennungen von Lipoiden an Kieselsäure -gehen auf. WEICKER (4), MANGOLD (5) und WAGNER und Mitarbeiter (6) zurück; weitere Arbeiten auf diesem Gebiet haben dabei ergeben, daß für die quantitative chromatographische Bestimmung der Lipoide die Dünnschicht gegenüber der Säule und dem Papier wesentliche Vorteile bietet. Eine Reihe von Autoren (4, 7-16) haben teils für qualitative Untersuchungen aber auch für quantitative dünnschichtchromatographische Bestimmungen von Phosphatiden und Sphingolipoiden Methoden ausgearbeitet. Die erhaltenen Ergebnisse lassen erkennen, daß der erzielte Trenneffekt bei geringerem Zeitaufwand gegenüber der Säule wesentlich besser ist. Die verschiedenen Methoden unterscheiden sich hauptsächlich in der Lipoidextraktion, der Aufarbeitung des Lipoidextraktes und der quantitativen Bestimmung der Lipoidfraktionen nach der chromatographischen Trennung, während die eigentlichen Chromatographie-Bedingungen nur geringfügig voneinander abweichen. Wir stellten uns deshalb die Aufgabe, die bisher nicht voll befriedigenden Teiloperationen - besonders die Lipoidextraktion und die quantitative Bestimmung der getrennten Lipoide näher zu untersuchen. Das Ziel war die Ausarbeitung einer möglichst einfachen, aber gut reproduzierbaren, speziell für die Lipoidanalyse im Serum geeigneten Routinetechnik.

\section{Methodik}

Es wird hier die ausführliche Beschreibung der Methode wiedergegeben, wie sie sich gestützt auf die Ergebnisse der nachfolgend beschriebenen Versuche als optimal erwiesen hat.
Lipoidextraktion (nach RENKONEN)

Das Serum oder Plasma soll möglichst frisch zur Analyse gelangen, da bei Lagerung auch bei $+2^{\circ}$ durch Hydrolyse der Lysophosphatidgehalt dauernd ansteigt. Wir verwendeten deshalb nur Proben, die höchstens 24 Stunden bei $2^{\circ}$ oder aber bei $-20^{\circ}$ aufbewahrt worden waren.

In einem Reagenzglas mit Schliffstopfen gibt man $1,0 \mathrm{~m} /$ Serum oder Plasma zu $4,3 \mathrm{~m} /$ Chloroform-Methanol (5:8) (sämtliche Reagenzien und Lösungsmittel „pro analysi“). Ohne zu bewegen wird das Gemisch 8 Min. stehen gelassen. Dann wird während 30 Sek. stark geschüttelt und weitere $5 \mathrm{Min}$. stehen gelassen. Es werden dann $3,7 \mathrm{~m} /$ Chloroform und $1,0 \mathrm{~m} / \mathrm{H}_{2} \mathrm{O}$ zugegeben, zehnmal umgeschwenkt und $10 \mathrm{Min}$. stehen gelassen. Dann wird während $15 \mathrm{Min}$. bei etwa $1500 \mathrm{~g}$ zentrifugiert. Dabei trennt sich die wäßrige Phase von der Chloroformphase, wobei sich das denaturierte Serumprotein als feste Schicht an der Grenzfläche ansammelt. Mittels einer an Vakuum angeschlossenen. Pipette wird die obere wäßrige Schicht vollständig abgesogen"und die Proteinschicht mit einem Spatel etwas auf die Seite geschoben. Die Chloroformphase, welche theoretisch ein Volumen von $6,0 \mathrm{ml}$ aufweist, wird sodann durch ein kleines Filter gegeben oder nach Abgießen erneut zentrifugiert. Für die weitere Verarbeitung steht in der Regel ein aliquotes klares Volumen von $3-5 \mathrm{~m} l$ zur Verfügung. Das aliquote Volumen (bei uns meist $4,0 \mathrm{ml}$ ) wird im Wasserbad bei $37^{\circ}$ unter Einblasen von $\mathrm{N}_{2}$ in einem tarierten Reagenzglas eingedampft, und der Rückstand über Nacht im Vakuumexsiccator über $\mathrm{P}_{2} \mathrm{O}_{5}$ getrocknet. Der Mittelwert des in der Dreifachbestimmung ermittelten Trockengewichtes auf $6,0 \mathrm{~m} l$ umgerechnet, ergibt den Gehalt von $1 \mathrm{~m} l$ Serum an Gesamtlipoiden.

Bestimmung der Gesamt-Phosphatide

Das getrocknete Totallipoid wird im gleichen Röhrchen mit Chloroform unter leichtem Erwärmen gelöst, sodaß eine Lösung von etwa $10 \mathrm{mg}$ Totallipoid $/ \mathrm{m} l$ entsteht. Diese Lösung wird sowohl für die Phosphorbestimmung wie auch für die Chromatographie verwendet.

$0,1 \mathrm{ml}$ dieser Lösung werden auf $3,5 \mathrm{~m} l$ mit Chloroform verdünnt und je $0,5 \mathrm{~m} l$ dieser Lösung werden in 3 Pyrex-Reagenzgläser abpipettiert. Das Lösungsmittel wird auf kleiner Flamme abgedampft. Die Röhrchen sind damit für die P-Bestimmung bereit; sie enthalten je etwa $1,5 \mu \mathrm{g} P$.

Phosphor-Bestimmung (nach SHIN modifiziert)

Pro Röhrchen gibt man $0,75 \mathrm{ml} \mathrm{H}_{2} \mathrm{SO}_{4} 10 \mathrm{~N} z \mathrm{zu}$ und erhitzt auf mittlerer Flamme bis alles Wasser abgetrieben ist. (Der Kondensationsring der $\mathrm{H}_{2} \mathrm{SO}_{4}$ muß bis zum oberen Rand des Reagenzglases gebracht werden.) Die Aufschlußlösung ist braun gefärbt. Nach Erkalten gibt man 1 Tropfen Perhydrol zu und erhitzt bis alles Wasser verdampft und die Lösung farblos geworden ist. Nach Erkalten 1 Tropfen Harnstoff 5-proz. zusetzen und wiederum er- 
hitzen, bis allcs Wasser ausgetricben ist. Zur abgekühlten Probe gibt man $4,35 \mathrm{~m} / \mathrm{H}_{2} \mathrm{O}$ und $0,2 \mathrm{~m} / 5$-proz. Ammonmolybdat $4 \mathrm{H}_{2} \mathrm{O}$ und $0,2 \mathrm{~m} /$ Reagenz nach Fisk-Subra-Row (17) $(6,85 \mathrm{~g}$ $\mathrm{Na}_{2} \mathrm{~S}_{2} \mathrm{O}_{5}$ werden in $50 \mathrm{~m} / \mathrm{H}_{2} \mathrm{O}$ bidest gelöst. 0,125 g Aminonaphtolsulfosäurc zugeben und kräftig schütteln bis nur noch geringe Trübung bleibt. Tropfenweises Zufügen ciner 30-proz. $\mathrm{Na}_{2} \mathrm{SO}_{3}$-Lösung bis die lösung völlig klar ist. Die Lösung ist im Dunkeln aufbewahrt höchstens 14 Tage haltbar). Die Röhrchen mit einem Totalvolumen von $5,0 \mathrm{ml}$ werden während $7 \mathrm{Min}$. in ein siedendes Wasserbad gestellt und anschließend 5 Min. im Eisbad gekïhlt. Die Extinktion wird innerhalb 20 Min. im Spektralphotometer bei $830 \mathrm{~m} \mu$ gegen Wasser gemessen. Gleichzeitig werden 3 Reagenzienblindwerte und drei P-Standards $(2 \mu \mathrm{g} \mathrm{P})$ mitgeführt (Stammlösung: 6,74 g Na NH $\mathrm{HPO}_{4} \cdot 4 \mathrm{H}_{2} \mathrm{O}$ p. a., Fa. Merck in $1000 \mathrm{~m} / \mathrm{H}_{2} \mathrm{O}$ bidest; P-Standard: Verdünnung 1:500 $=2 \mu \mathrm{g}$ $\mathrm{P} / \mathrm{m} /)$.

\section{Bereclonung}

Die Mittelwerte der Extinktionen werden in der Eichkurve auf $\mu \mathrm{g} P$ umgerechnet und müssen mit 7 und 25 multipliziert werden (durchschnittlicher P-Gehalt der Phosphatide etwa 4\%). Man erhält sodann das Gewicht der Phosphatide in $1 \mathrm{mg}$ Totallipoid.

Quantitative Dünnschichtchromatographie

Vorberitung der Scbicbten

$25 \mathrm{~g}$ Kieselgel HR reinst ( $\mathrm{Fa}$. Merck) werden in einem SchliffErlenmeyerkolben mit $50 \mathrm{~m} / \mathrm{H}_{2} \mathrm{O}$ versetzt und während $30 \mathrm{Min}$. stark geschüttelt. Dann wird der offene Kolben in einem Vakuumexsiccator zur Entfernung der Luftbläschen kurz evakuiert. Das Gel wird dann mit einem Streichgerät auf 5 entfetteten Platten $20 \times 20 \mathrm{~cm}$ in einer Schichtdicke von $0,25 \mathrm{~mm}$ ausgestrichen und an der Luft getrocknet. Unmittelbar vor Gebrauch werden die Platten während $90 \mathrm{Min}$. bei $110^{\circ}$ aktiviert.

\section{Auftragen der Proben}

Pro Platte können bis zu acht Proben $15 \mathrm{~mm}$ oberhalb des unteren Randes strichförmig aufgetragen werden. Am günstigsten ist jedoch die Anordnung mit 6 Proben, je auf einer Länge von $1 \mathrm{~cm}$ aufgetragen, mit einem Abstand von $2 \mathrm{~cm}$ zwischen den Proben. Dic Auftragstellen werden mit einer Nadel auf der Platte markiert, wonach mit einer Milkropipette von jedem Serum 2 Proben à $0,1 \mathrm{~m} l$ aufgetragen werden (je 2 Proben von 2 der ursprünglichen 3 Lipoidextrakte). Die Auftragung kann am besten tropfenweise (Tropfen à etwa $5 \mu l$ entlang der Auftraglinie) bewerkstelligt werden, wobei jeweils mit dem Gebläse getrocknet wird. Die Schicht soll bei der Auftragung nicht verletzt werden.

\section{Cliromatographie}

Laufmittel: Chloroform-Methanol- $\mathrm{H}_{2} \mathrm{O}$ (14:6:1) (11). Die mit Filterpapier ausgekleideten Kammern sollen mindcstens 4 Stdn. mit dem Laufmittel $(100 \mathrm{ml})$ zur Sättigung der Gasphase stehen gelassen werden. Die Platten werden sodann während 30-45 Min. chromatographiert, bis die Lösungsmittelfront eine Höhe von $16 \mathrm{~cm}$ erreicht hat. Die Platten werden dann sofort unter dem Gebläse getrocknet und anschließend in eine Kammer gebracht, in welcher kurz vorher einige Kristalle Jod in einem erhitzten Tiegel verdampft wurden. Nach etwa 15 Min. kann die Platte herausgenommen werden und die 4 unverkennbaren Flecken für (von oben nach unten) Kephalin, Lecithin, Sphingomyelin (Doppellinie) und Lysolecithin werden mit einer Nadel etwa $2 \mathrm{~mm}$ außerhalb der Farbgrenze des Jods punktförmig umrandet (Abb. 1).

Sehr gute Trennungen erhält man auch mit einer von JATZKEwITZ (11) veröffentlichten 2-Stufentechnik. Dic Platten werden dabei zuerst nach der oben aufgeführten Vorschrift chromatographiert, dann aber nur während $5 \mathrm{Min}$. an der Luft antrocknen gelassen und anschließend in einer zweiten Kammer mit n-Propanol/12,5-proz. $\mathrm{NH}_{3}(4: 1)$ bis zu einer Höhe von $10 \mathrm{~cm}$ erneut chromatographiert (Abb. 2).

\section{Eluierung der Phospholipoidfraktionen}

Die auf der Platte angezeichneten Fraktionen werden einzeln mit dem Spatel auf ein Pergamentpapier abgekratzt, in ein Reagenzglas

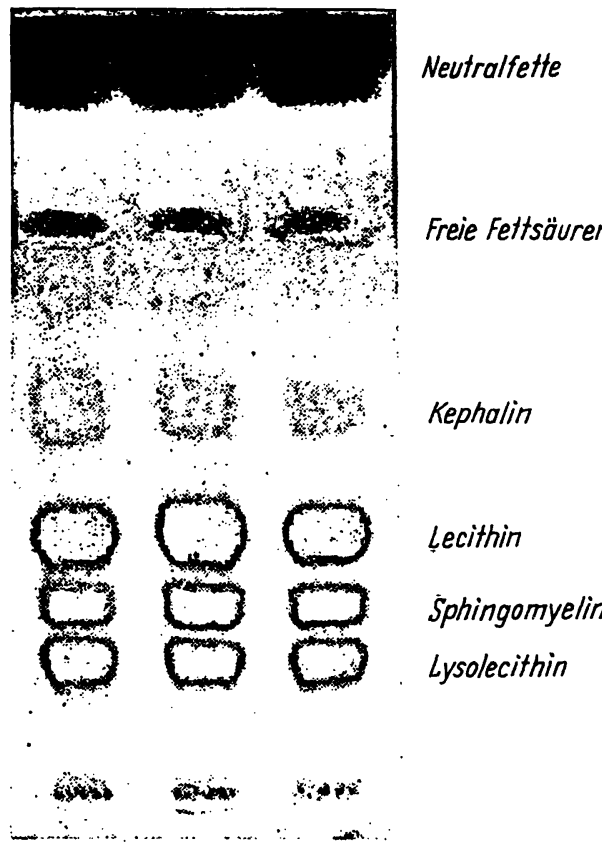

Abb. 1

Dünnschichtchromatogramm eines Lipoidextraktes aus Serum; Auftragmenge: $1 \mathrm{mg}$

Schicht: Kieselgel HR reinst Merck; Solvens: Chloroform/Methanol/ $\mathrm{H}_{2} \mathrm{O} 14: 6: 1$; Laufstrecke $16 \mathrm{~cm}$; Färbung: Joddämpfe
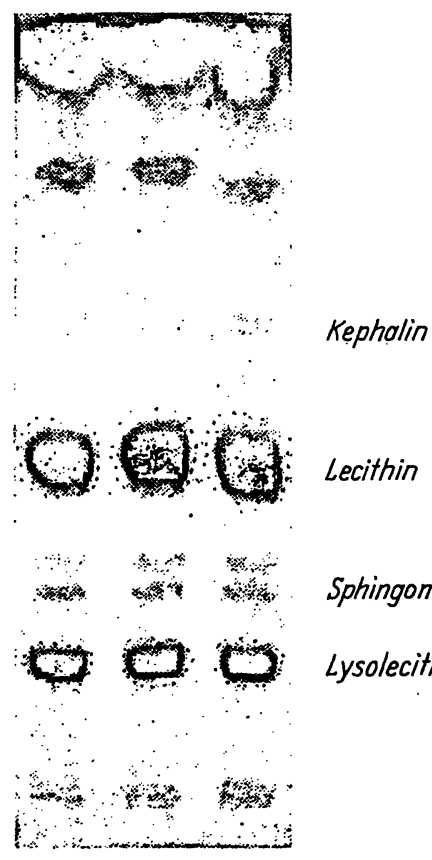

Abb. 2

Dünnschichtchromatogramm eines Lipoidzxtraktes aus Serum; Auftragmenge: $1 \mathrm{mg}$ Schicht: Kieselgel HR reinst Merck; Solvens: a) Chloroform/Methanol/
$\mathrm{H}_{2} \mathrm{O}$ 14:6:1; b) n-Propanol/12,5\% $\mathrm{NH}_{3}$ 4:1; Laufstrecke: a) $16 \mathrm{~cm}$ b) $10 \mathrm{~cm}$

gebracht und anschließend mit $5 \mathrm{ml}$ folgender Lösungsmittelgemische versetzt: Kephalin: Chloroform-Methanol (2:1), Lecitbin und Sphingomyelin: Chloroform-Methanol (1:1) und Lysolecitbin: Methanol- $\mathrm{H}_{2} \mathrm{O}(1: 1)$. Die Röhrchen werden 15 Min. im Wasserbad bei $45^{\circ}$ durch gelegentliches Rühren mit einem eingepaßten Glasstab extrahiert und anschließend $5 \mathrm{Min}$. bei etwa $1500 \mathrm{~g}$ zentrifugiert. Der erste Extrakt wird abgegossen und das Kieselgelsediment wird nochmals zweimal auf dieselbe Art extrahiert. Die gesammelten Extrakte werden sodann im Wasserbad bei $60^{\circ}$ durch Einblasen von $\mathrm{N}_{2}$ zur Trockene gebracht. Die anschließende PBestimmung kann in densclben Röhrchen durchgeführt werden. 


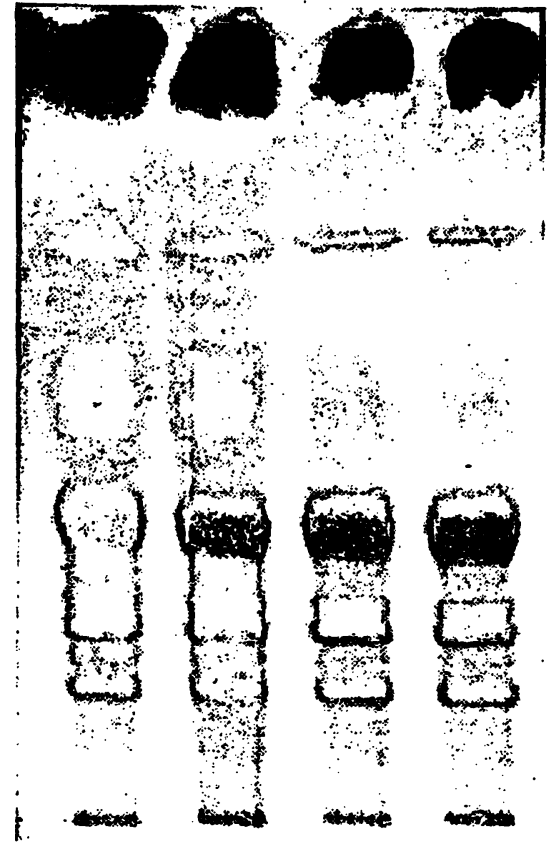

Abb. 3

Dünnschichtchromatogramm von 2 Lipoidextrakten aus demselben Serum wie Abbildung 2

Schicht: Kieselgel HR reinst Merck; Solvens a) Chloroform/Methanol/ $\mathrm{H}_{2} \mathrm{O} 14: 6: 1 ;$ b) n-Propanol/12,5\% $\mathrm{NH}_{3} 4: 1$; Färbung: Joddämpfe

\section{Phosphor-Bestimmung}

Man verfährt genau gleich wie vorher beschrieben. Für die Berechnung lcgt man folgende Faktoren zugrunde: Kephalin, Lecithin, Sphingomyelin: 25 und Lysolecithin: 16,7. Der errechnete Wert ergibt die Menge der entsprechenden Phospholipoidfraktion pro $1 \mathrm{mg}$ Gesamtlipoid (aufgetragene Menge).

Bcispiel (Resultate s. Tab. 1-3)

Lipoidbestimmung in einem normalen menscblicben Serum

a) Lipoidextraktion von 3 Proben à $1 \mathrm{ml}$.

Je $4 \mathrm{ml}$ der Chloroformphase eingetrocknet und gewogen: 4,$70 ; 4,61$ und $4,60 \mathrm{mg} / 4 \mathrm{ml}$, umgerechnet auf die Gesamtmenge des Chloroforms von $6 \mathrm{~m} /$ ergibt entsprechend: 7,05;6;92 und $6,90 \mathrm{mg}$ Gesamtlipoide/ $\mathrm{m} l$ Serum.

Mittelwert für Gesamtlipoide: $696 \mathrm{mg} \%$

b) Phosphatide

$\mathrm{Zu}$ Röhrchen 2 und 3 werden je $0,46 \mathrm{~m} /$ Chloroform gegeben, wobei eine klare Lösung entsteht. $0,1 \mathrm{~m} l$ dieser Lösungen werden entnommen und auf $3,5 \mathrm{~m} / \mathrm{mit}$ Chloroform ergänzt. Davon werden dreimal je $0,5 \mathrm{~m} l$ abpipettiert, eingetrocknet und zur P-Bestimmung gebracht (Tab. 1).

c) Chromatographie

Auftragmengen: $2 \mathrm{mal}$ je $0,1 \mathrm{~m} l$. Chromatogramm: Abbildung 3 .

\section{Ergebnisse}

Extraktion der Lipoide aus Serum oder Plasma De Iongh und van Pelt (18) haben kürzlich gezeigt, $\mathrm{da} ß$ die von Folch und Mitarbeiter (19) für Gewebe ausgearbeitete Lipoid-Extraktion auch für Serum bessere Resultate zeigt, als die im allgemeinen übliche Extraktion nach BLOOR (20). RENKONEN und Mitarbeiter (21) haben nun diese Methode in Anlehnung an die Arbeitstechnik von BLIG und DYYER '(22) modifiziert und ein außerordentlich einfaches Extraktionsverfahren ausgearbeitet, welches bezüglich Genauigkeit dem FolCHschen Verfahren kaưm nachsteht, dafür aber eine wesentliche Vereinfachung und Verkürzung des Extrak-

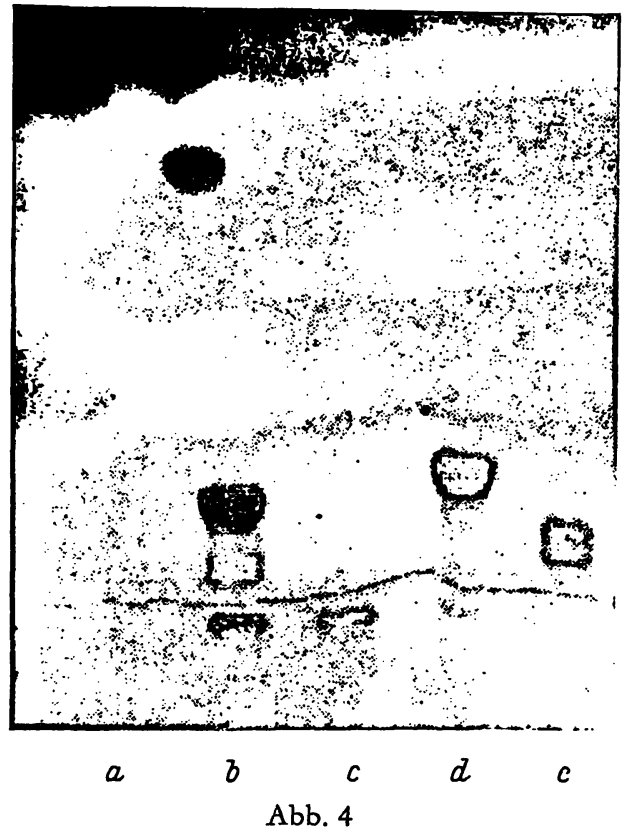

Dünnschichtchromatogramm mit Jod gefärbt; Laufmittel nach JATZKEWITZ
a) $5 \mu \mathrm{g}$ Kephalin
b) $600 \mu \mathrm{g}$ Serumlipoidextrakt
d) $100 \mu \mathrm{g}$ Lecithin
c) $20 \mu \mathrm{g}$ Lysolecithin
e) $20 \mu \mathrm{g}$ Sphingomyelin

tions-Vorganges darstellt. Wir haben in einer Reihe von Versuchen an einigen Sera die Methode von BLoor (20), FolCh und Mitarbeitern (19) und RENKonen und Mitarbeitern (21) vergleichend durchgeführt und können bestätigen, daß die nach RENKONEN gefundenen Werte gut reproduzierbar sind und der Folch'schen Methode weitgehend entsprechende Resultate ergeben.

1 Normalserum und 2 hyperlipämische Sera wurden in je 10 Versuchen nach 3 Methoden extrahiert (BLOoR, FolCH und RENKONEN) und anschließend gravimetrisch das Gesamtlipoid und mittels einer Phosphor-Bestimmung der Phosphatid-Anteil bestimmt.

\section{Extraktion nacb. For $\mathrm{CH}$}

$1 \mathrm{~m} l$ Serum wird in $19 \mathrm{~m} l$ Chloroform-Methanol (2:1) eingegeben und während $3 \mathrm{Min}$. stark geschüttelt. Man läßt 30 Min. stehen und filtriert die Ausfällung ab. $\mathrm{Zu}$ $10 \mathrm{~m} l$ der klaren Lösung gibt $\operatorname{man} 2 \mathrm{ml} \mathrm{H}_{2} \mathrm{O}$ und schüttelt stark während 30 Sek. Die Emulsion wird bei $1500 \mathrm{~g}$ während 20 Min. zentrifugiert, wonach die obere Phase möglichst weitgehend abgesogen wird. Man gibt nochmals $2 \mathrm{~m} l$,theoretische obere Phase" zu (Chloroform-Methanol- $\left.\mathrm{H}_{2} \mathrm{O}(3: 48: 47)\right)$ schüttelt gut durch und zentrifugiert erneut, um die obere Schicht abzusaugen. Die Chloroformphase wird sodann unter $\mathrm{N}_{2}$ bei $37^{\circ}$ eingetrocknet und gewogen. Auflösen des Lipoides in Chloroform und Bestimmung des Phosphors im aliquotem Teil.

\section{Extraktion nach BLOOR}

$1 \mathrm{~m} l$ Serum wird tropfenweise in $19 \mathrm{~m} /$ einer siedenden Mischung von Äthanol-Äther (3:1) gegeben und während 1 Min. unter Rühren weiter gekocht. Die Mischung wird durch ein Papierfilter filtriert und das Filter mit 
Tab. 1

Resultat der Phosphatidbestimmung; Mittelwert für Gesamtphosphatide $197 \mathrm{mg} \%$ oder 28,4\% Phosphatide vom Gesamtlipoid

\begin{tabular}{|c|c|c|c|c|c|c|}
\hline Probe & Extinktion & Mittelwert & $\underset{\text { pro Röhrchen }}{\mu \mathrm{g} \text { Phosphor }}$ & Gesamt & $\underset{\text { Gesamt-Phosphatide }}{\mathrm{mg}}$ & $\begin{array}{l}\text { \% Phosphatide } \\
\text { vom Gesamtlipoid }\end{array}$ \\
\hline $\begin{array}{l}\text { Standard } \\
(2 \mu \mathrm{gP})\end{array}$ & $\begin{array}{l}0,362 \\
0,365 \\
0,364\end{array}$ & 0,3637 & - & - & - & - \\
\hline Probe 2 & $\begin{array}{l}0,297 \\
0,301 \\
0,302\end{array}$ & 0,300 & 1,645 & $\left.79,5^{1}\right)$ & 1,987 & 28,7 \\
\hline Probe 3 & $\begin{array}{l}0,301 \\
0,299 \\
0,297\end{array}$ & 0,299 & 1,650 & $\left.77,9^{2}\right)$ & 1,945 & 28,2 \\
\hline
\end{tabular}

1) Umrechnungsfaktor $f=\frac{3,5}{0,5} \cdot \frac{0,46}{0,1} \cdot \frac{6,92}{4,61}=48,336$

2) Uinrechnungsfaktor $f=\frac{3,5}{0,5} \cdot \frac{0,46}{0,1} \cdot \frac{6,90}{4,60}=48,300$

Tab. 2

Resultate der Phosphor-Bestimmung der Fraktionen

\begin{tabular}{|c|c|c|c|c|c|c|}
\hline Fraktion & Extinktion ${ }^{1}$ ) & Mittelwert & pro Röhrchen & or ${ }_{\text {Gesamt }}^{2}$ ) & $\begin{array}{l}\text { Gesamt- } \\
\text { Phosphatid } \\
\text { in } \mathrm{mg}^{3} \text { ) }\end{array}$ & $\begin{array}{l}\% \text { Phosphatid } \\
\text { vom Gesamt- } \\
\text { Phosphatid }\end{array}$ \\
\hline \multirow{2}{*}{$\begin{array}{l}\text { Standard } \\
\qquad(2 \mu \mathrm{gP})\end{array}$} & 0,361 & & & & & \\
\hline & $\begin{array}{l}0,365 \\
0,364\end{array}$ & 0,3633 & & & & \\
\hline \multicolumn{7}{|l|}{ Kephalin } \\
\hline \multirow{2}{*}{$\begin{array}{r}\text { Probe } 2 \mathrm{a} \\
2 \mathrm{~b} \\
3 \mathrm{a} \\
3 \mathrm{~b}\end{array}$} & $\left.\begin{array}{l}0,026 \\
0,053\end{array}\right\}$ & 0,0395 & 0,218 & 1,50 & 37,5 & 1,89 \\
\hline & $\left.\begin{array}{l}0,039 \\
0,056\end{array}\right\}$ & 0,0475 & 0,262 & 1,81 & 45,2 & 2,32 \\
\hline \multicolumn{7}{|l|}{ Lecithin's) } \\
\hline \multirow{2}{*}{$\begin{array}{r}\text { Probe } 2 \mathrm{a} \\
2 \mathrm{~b} \\
3 \mathrm{a} \\
3 \mathrm{~b}\end{array}$} & $\left.\begin{array}{l}0,618 \\
0,606\end{array}\right\}$ & 0,612 & 3,37 & 46,4 & 1160 & 58,4 \\
\hline & $\left.\begin{array}{l}0,605 \\
0,591\end{array}\right\}$ & 0,598 & 3,30 & 45,6 & 1140 & 58,7 \\
\hline \multirow{3}{*}{$\begin{array}{c}\text { Sphingomyelin } \\
\text { Probe } 2 \mathrm{a} \\
2 \mathrm{~b} \\
3 \mathrm{a} \\
3 \mathrm{~b}\end{array}$} & & & & & & \\
\hline & $\left.\begin{array}{l}0,355 \\
0,349\end{array}\right\}$ & 0,352 & 1,94 & 13,4 & 335 & 16,9 \\
\hline & $\left.\begin{array}{l}0,358 \\
0,384\end{array}\right\}$ & 0,371 & 2,04 & 14,1 & 353 & 18,2 \\
\hline \multicolumn{7}{|l|}{ Lysolecithin } \\
\hline \multirow{2}{*}{$\begin{array}{r}\text { Probe } 2 \mathrm{a} \\
2 \mathrm{~b} \\
3 \mathrm{a} \\
3 \mathrm{~b}\end{array}$} & $\left.\begin{array}{l}0,281 \\
0,319\end{array}\right\}$ & 0,300 & 1,65 & 11,4 & 190 & 9,57 \\
\hline & $\left.\begin{array}{l}0,280 \\
0,272\end{array}\right\}$ & 0,276 & 1,52 & 10,4 & 173 & 8,90 \\
\hline
\end{tabular}

1) Der Reagenzienblindwert ergibt sich durch Auskratzen einer ungefähr der Größe der Fraktionen entsprechenden Region Kieselgel in einem Gebiet, das keine Färbung mit Jod aufweist.

2) Der Gesamtphosphor errechnet sich nach der aliquoten Auftragsmenge: $F=\frac{0,46}{0,1} \cdot \frac{6}{4}=6,9$.

2) Umrechnungsfaktoren betragen für Kephalin, Lecithin und Sphingomyelin 25, für Lysolecithin 16,7.

) Bei den Proben 2 rechnet man die \%-Werte auf die Gesamtphosphatidmenge von 1,987 mg, bei den Proben 3 auf 1,945 mg

5) Vor der Veraschung wurden die Proben durch erneutes Aufnehmen in 1,0 ml Chloroform und Abpipettieren von 0,5 ml auf die Hälfte reduziert, da sonst die Extinktionen den Wert von 1,0 übersteigen.

Tab. 3

Endresultat der Lipoidzusammensetzung

\begin{tabular}{lcc}
\hline Gesamtlipoide & $\begin{array}{c}696 \mathrm{mg} \% \\
28,4 \%\end{array}$ & $\begin{array}{c}\text { Variationskoeffizient }{ }^{2} \text { ) } \\
\pm 1,2 \%\end{array}$ \\
$\begin{array}{l}\text { Anteil Phosphatide } \\
\text { Phosphatide in \% vom Gesamt-Phosphatid }\end{array}$ & \\
\hline Kephalin & 2,1 & $\pm 34 \%$ \\
Lecithin & 58,6 & $\pm 2,4 \%$ \\
Sphingomyelin & 17,5 &, $\pm 6 \%$ \\
Lysolecithin & 9,2 & $\pm 9 \%$ \\
Restliche nicht bestimmte & 12,6 & \\
Phosphatide & & \\
(Differenz zu 100\%) & & \\
\hline
\end{tabular}

2) Dieser Wert $\left(-\frac{S \cdot 100}{\bar{x}}\right)$ ergab sich aus den statistischen Berechnungen, (s. Abb. 6-11).

insgesamt $8 \mathrm{~m} l$ des heißen Lösungsmittels nachgewaschen. Der Extrakt wird unter Vakuum bei $60^{\circ}$ zur Trockene eingedampft, in etwa 2-3 $\mathrm{ml}$ Chloroform gelöst, erneut filtriert, und das Filter mit etwa 2-3 ml Chloroform nachgewaschen. Die Lösung wird unter $\mathrm{N}_{2}$ bei $40^{\circ}$ zur 'Trockene gebracht und gewogen. Phosphor-Bestimmung im aliquoten Teil einer Chloroformlösung des getrockneten Gesamtlipoides.

\section{Extraktion nach RENKONEN}

Analog der unter „Methodik“ beschriebenen Lipoidextraktion.

\section{Resultate}

Die nach den 3 Methoden durchgeführten Bestimmungen (s. Tab. 4) bestätigten, daß die ReNkonen-Technik trotz ihrer Einfachheit Werte liefert, welche den nach FolcH erhaltenen Resultaten sehr angenähert sind. Auch die Streuung der Resultate ist bei beiden Methoden im gleichen Rahmen. Entgegen den Resultaten vergleichender Untersuchungen von JEstrng und BANG (23) erhielten wir mit der BLOoR-Technik durchweg höhere Werte als mit den beiden anderen Methoden. Sowohl der Gesamtlipoidgehalt wie der Phosphorgehalt liegen bei der BLooR-Technik etwa 11-19\% höher, was wir auf das Fehlen eines Auswaschprozesses bei dieser Methode und damit auf die Mitbestimmung von NichtLipoiden zurückführen möchten. Für Routine-Lipoidextraktionen aus Serumproben kann somit die RENKONEN-Technik ohne weiteres empfohlen werden. 
Tab. 4

Vergleich der Lipoidextraktionsmethoden nach RENKONEN, FOLCH und BLOOR

\begin{tabular}{|c|c|c|c|c|c|c|}
\hline \multirow[b]{2}{*}{ Serumprobe } & \multicolumn{2}{|c|}{ Methode RENKONEN } & \multicolumn{2}{|c|}{ Methode FOLCH } & \multicolumn{2}{|c|}{ Methode BLOOR } \\
\hline & $\begin{array}{l}\text { Gesamtlipoid } \\
\text { mg\% }\end{array}$ & $\begin{array}{l}\text { Phosphatide } \\
\%\end{array}$ & $\begin{array}{l}\text { Gesamtlipoid } \\
\text { mg\% }\end{array}$ & $\begin{array}{l}\text { Phosphatide } \\
\%\end{array}$ & $\begin{array}{c}\text { Gesamtlipoid } \\
\text { mg \% }\end{array}$ & $\begin{array}{c}\text { Phosphatide } \\
\%\end{array}$ \\
\hline $\begin{array}{l}\text { Mittelwert } \\
\text { Standardabweichung }\end{array}$ & $\begin{array}{l}681 \\
686 \\
697 \\
720 \\
684 \\
664 \\
701 \\
675 \\
699 \\
693 \\
690 \\
15,61\end{array}$ & $\begin{array}{c}30,2 \\
30,1 \\
31,0 \\
29,8 \\
30,1 \\
30,5 \\
30,9 \\
31,0 \\
30,3 \\
31,1 \\
30,5 \\
0,446\end{array}$ & $\begin{array}{l}661 \\
671 \\
688 \\
662 \\
670 \\
656 \\
680 \\
682 \\
679 \\
669 \\
672 \\
10,32\end{array}$ & $\begin{array}{c}28,6 \\
27,8 \\
29,1 \\
29,6 \\
28,4 \\
27,9 \\
28,0 \\
29,5 \\
29,4 \\
28,6 \\
28,7 \\
0,679\end{array}$ & $\begin{array}{c}740 \\
716 \\
784 \\
750 \\
776 \\
725 \\
738 \\
762 \\
780 \\
781 \\
755 \\
24,9\end{array}$ & $\begin{array}{c}32,1 \\
29,9 \\
30,5 \\
31,4 \\
32,0 \\
30,1 \\
31,3 \\
32,4 \\
31,1 \\
31,5 \\
31,2 \\
0,845\end{array}$ \\
\hline Variationskoeffizient in \% & $\pm 2,3 \%$ & $\pm 1,5 \%$ & $\pm 1,5 \%$ & $\pm 2,3 \%$ & $\pm 2,3 \%$ & $\pm 2,7 \%$ \\
\hline $\begin{array}{l}\text { Mittelwert } \\
\text { Standardabweichung }\end{array}$ & $\begin{array}{c}1201 \\
1181 \\
1223 \\
1169 \\
1205 \\
1241 \\
1215 \\
1251 \\
1237 \\
1181 \\
1210 \\
27,9\end{array}$ & $\begin{array}{c}18,1 \\
18,5 \\
17,9 \\
18,1 \\
18,9 \\
18,2 \\
18,3 \\
17,6 \\
18,7 \\
17,8 \\
18,2 \\
0,405\end{array}$ & $\begin{array}{l}1227 \\
1173 \\
1162 \\
1205 \\
1215 \\
1190 \\
1154 \\
1175 \\
1210 \\
1207 \\
1192 \\
24,6\end{array}$ & $\begin{array}{c}17,7 \\
17,2 \\
18,2 \\
18,2 \\
18,4 \\
17,8 \\
17,3 \\
17,4 \\
17,9 \\
17,8 \\
17,8 \\
0,404\end{array}$ & $\begin{array}{c}1372 \\
1410 \\
1352 \\
1467 \\
1422 \\
1488 \\
1405 \\
1436 \\
1428 \\
1416 \\
1420 \\
40,8\end{array}$ & $\begin{array}{c}18,4 \\
17,9 \\
17,9 \\
19,1 \\
19,4 \\
18,3 \\
17,6 \\
18,0 \\
19,3 \\
19,0 \\
18,5 \\
0,657\end{array}$ \\
\hline Variationskoeffizient in $\%$ & $\pm 2,3 \%$ & $\pm 2,2 \%$ & $\pm 2,1 \%$ & $\pm 2,2 \%$ & $\pm 2,8 \%$ & $\pm 3,5 \%$ \\
\hline $\begin{array}{l}\text { Mittelwert } \\
\text { Standardabweichung }\end{array}$ & $\begin{array}{l}5850 \\
6200 \\
6090 \\
6070 \\
5980 \\
5980 \\
6030 \\
5920 \\
5940 \\
6130 \\
6020 \\
105,7\end{array}$ & $\begin{array}{l}6,51 \\
6,33 \\
6,36 \\
6,50 \\
6,45 \\
6,40 \\
6,39 \\
6,48 \\
6,34 \\
6,53 \\
6,43 \\
0,0724\end{array}$ & $\begin{array}{l}6210 \\
6020 \\
5960 \\
6260 \\
6070 \\
6100 \\
5910 \\
5990 \\
6130 \\
6160 \\
6080 \\
112,4\end{array}$ & $\begin{array}{l}6,34 \\
6,11 \\
6,21 \\
6,06 \\
6,40 \\
6,44 \\
6,22 \\
6,24 \\
6,16 \\
6,32 \\
6,25 \\
0,1238\end{array}$ & $\begin{array}{c}7310 \\
7250 \\
7455 \\
7080 \\
7120 \\
7020 \\
7240 \\
7410 \\
7280 \\
7230 \\
7240 \\
137,5\end{array}$ & $\begin{array}{l}7,28 \\
7,62 \\
7,51 \\
7,84 \\
7,31 \\
7,26 \\
7,66 \\
7,43 \\
7,84 \\
7,28 \\
7,50 \\
0,228\end{array}$ \\
\hline Variationskoeffizient in $\%$ & $\pm 1,7 \%$ & $\pm 1,12 \%$ & $\pm 1,8 \%$ & $\pm 1,9 \%$ & 土 $1,9 \%$ & $\pm 3,0 \%$ \\
\hline
\end{tabular}

\section{Phosphor-Bestimmung}

Für die quantitative Erfassung besonders der nur in geringen Mengen vorliegenden Kephalinfraktion muß von einer Mikromethode zur Phosphorbestimmung eine Mindestempfindlichkeit von $0,1 \mu \mathrm{g} P$ pro Probe verlangt werden. Wir hofften, dieses Ziel unter Verwendung einer Methode zu erreichen, bei der die Veraschung der Probe bei Anwesenheit des Kieselgels erfolgt; das Trägermaterial wird bei dieser Methodik erst unmittelbar vor dem Photometrieren des entwickelten Farbstoffs abgetrennt. Wir folgten dabei Vorschriften von DotzakI und ZIEve (9), welche nach BartLETt (24) arbeiten, sowie von RoBINson und PhILirps (12), welche eine Modifikation der BARTLETT-Technik angeben. Bei beiden Methoden, wie auch bei einer äußerst empfindlichen Modifikation der BARTLETT-Technik, welche SHIN (25) beschrieben hat, wurde aber eine eindeutige Störung der Resultate durch das Kieselgel HR beobachtet. Christian und Mitarbeiter (8) fanden nach der Methode von DorzakI und ZIEvE (9) Blindwerte für Kieselgel allein von 0,050 Extinktion pro mg Kieselgel, was wir größenordnungsmäßig bestätigen können. Die Streuung dieser Werte war jedoch bei uns sehr groß und führte speziell bei den Kephalinwerten zu Fehlerbreiten von mehr als $\pm 100 \%$.

Wir verließen deshalb diese mit vielen Schwierigkeiten verbundene direkte Veraschung des Kieselgels und wandten uns der Extraktion der Phospholipoidfràktionen aus dem ausgekratzten Kieselgel zu. Die ersten Versuche wurden mit reinen Phospholipoidfraktionen ${ }^{1}$ ) durchgeführt, welche wir auf einer Platte wie in der Vorschrift unter „Methodik“ angegeben, verarbeiteten. Als. Extraktionslösung verwendeten wir verschiedene Gemische von Chloroform und Methanol, wobei das ausgekratzte Kieselgel jeweils dreimal mit $5 \mathrm{~m} l$ des Lösungsmittels wie unter "Methodik“ angegeben, extrahiert wurde. Die höchsten Extraktionsausbeuten erhielten wir dabei mit folgenden Mischungen:

Kephalin: Chloroform-Methanol (2:1)

Lecithin: Chloroform-Methanol (1:1)

Sphingomyelin: Chloroform-Methanol (1:1)

Lysolecithin: Methanol- $\mathrm{H}_{2} \mathrm{O}(1: 1)$

Ein Kontrollversuch mit einem Serumlipoidextrakt, welchem ein Gemisch der reinen Testphosphatide mit bekanntem Gehalt beigefügt wurde, ergab folgendes Resultat (s. Tab. 5):

Serumlipoid in Chloroform: P-Gehalt $125 \mu \mathrm{g} \mathrm{P} / \mathrm{m} l$ Auftragmenge: $50 \mu \mathrm{l}(6,25 \mu \mathrm{g} \mathrm{P})$

Testgemisch in Chloroform: P-Gehalt $105 \mu \mathrm{g} \mathrm{P} / \mathrm{ml}$ Kephalin $10 \mu \mathrm{g} \mathrm{P} / \mathrm{m} l$, Lecithin $50 \mu \mathrm{g} \mathrm{P} / \mathrm{ml}$ Sphingomyelin $30 \mu \mathrm{g} \mathrm{P} / \mathrm{m} l$, Lysolecithin $15 \mu \mathrm{g} \mathrm{P} / \mathrm{m} l$ Auftragmenge $30 \mu l$ Testgemisch zusätzlich zu $50 \mu l$ Serumlipoidextrakt.

1) Lecithin und Kephalin der Fa. Sigma wurden chromatographisch an einer Kieselsäure-Kolonne gereinigt. Sphingomyelin verdanken wir Herrn Prof. KLENK, Köln. Lysolecithin erhiclten wir durch Einwirkung von Pankreas-Phospholipase A auf Lecithin und nachträgliche chromatographische Reinigung. 
Tab. 5

Ausbeuteversuch nach Zusatz eines Testgemisches von reinen Phosphatiden:

gefundene Phosphorwerte nach Chromatographie und Extraktion des Kieselgels

\begin{tabular}{|c|c|c|c|c|c|}
\hline & $\begin{array}{c}\text { Serumlipoid } \\
\text { Mg P gefunden }\end{array}$ & $\begin{array}{c}\text { Serumlipoid }+ \text { Testgemisch } \\
\mu \mathrm{g} P \text { gefunden }\end{array}$ & $\mu \mathrm{g}$ P Differenz & Theoretisch & Ausbeute (\%) \\
\hline Kephalin & $\left.\begin{array}{l}0,13 \\
0,14 \\
0,13\end{array}\right\} 0,133$ & $\left.\begin{array}{l}0,42 \\
0,40 \\
0,40\end{array}\right\} 0,413$ & 0,28 & 0,30 & 93 \\
\hline Lecithin & $\left.\begin{array}{l}3,65 \\
3,71 \\
3,62\end{array}\right\} 3,66$ & $\left.\begin{array}{l}5,18 \\
5,10 \\
5,17\end{array}\right\} 5,15$ & 1,49 & 1,50 & 99 \\
\hline Sphingomyelin & $\left.\begin{array}{l}1,26 \\
1,21 \\
1,25\end{array}\right\} 1,24$ & $\left.\begin{array}{l}2,10 \\
2,02 \\
2,08\end{array}\right\} 2,07$ & 0,83 & 0,90 & 92 \\
\hline Lysolecithin & $\left.\begin{array}{l}0,56 \\
0,60 \\
0,55\end{array}\right\} 0,57$ & $\left.\begin{array}{l}0,97 \\
0,99 \\
0,96\end{array}\right\} 0,97$ & 0,40 & 0,45 & 89 \\
\hline Total & $=90 \%^{5,60}$ & & 3,00 & 3,15 & 95 \\
\hline
\end{tabular}

Der Versuch zeigt, daß im Rahmen der Fehlerbreite der Methodik die zugegebenen Phosphatide nach der Extraktion meist zu mehr als $90 \%$ wiedergefunden werden. Die Ausbeute von $90 \%$ bei den Serumlipoiden allein beruht zum Teil darauf, daß einige weitere allerdings nur in geringfügiger Menge vorkommende Phosphatide wie z. B. Phosphatidsäure, Cardiolipin und Inositphosphatide gar nicht zur Bestimmung gelangen. Für die P-Bestimmung in den aus dem Kieselgel extrahierten Phosphatid-Fraktionen verwendeten wir eine Modifikation der einerseits von BARTLETT (24) und andererseits von SHIN (25) beschriebenen „Heatingmethod". Im Gegensatz zur ursprünglichen Frsk-SuBBARow (17)-Methode erhitzen diese Autoren das Reaktionsgemisch unmittelbar nach Zusatz des ReduktionsReagenzes während $7 \mathrm{Min}$. bei $100^{\circ}$ und erzielen dabei eine etwa 20fache Erhöhung der Extinktion. Unsere Modifikation folgt weitgehend den Bedingungen von Bartletr (24) mit Ausnahme der Aufschlußbedingungen. Durch Erhöhung des Schwefelsäurezusatzes von $0,5 \mathrm{~m} l$ auf $0,75 \mathrm{~m} l$ pro Röhrchen bei der Veraschung konnten wir verhindern, $\mathrm{da} ß$ bei der Veraschung höherer Lipoidmengen eine Farbabschwächung wegen zu niedriger $\mathrm{H}_{2} \mathrm{SO}_{4}$-Konzentration eintrat. (Ohne Einbuße der Farbausbeute wegen zu hoher Säuremenge könnten der Probe bis zu $0,85 \mathrm{~m} / \quad \mathrm{H}_{2} \mathrm{SO}_{4}$ zugefügt werden). Im weiteren haben wir anstelle einer total $4 \frac{1}{2}$ stündigen Aufschlußzeit bei $150-160^{\circ}$, wie dies BartLETt vorschreibt, vorgezogen, jede Probe auf dem Bunsenbrenner aufzuschließen, wobei pro Stunde etwa 15 bis 20 Röhrchen bearbeitet werden können.

\section{Identifizierung}

Bei Anwendung der unter „Methodik“ aufgeführten Bedingungen für die Dünnschichtchromatographie ist es ohne weiteres möglich, die Banden eindeutig zuzuordnen. Wir haben mit Hilfe von Testsubstanzen die Identifizierung durchgeführt und konnten dabei feststellen, daß bei der Färbung mit Jod eine falsche $\mathrm{Zu}$ ordnung der Fraktionen kaum möglich ist. Ejnige Schwierigkeiten kann allerdings das Kephalin bereiten, indem wohl die Bande eindeutig feststellbar ist, jedoch die Grenzen der Fraktion nicht immer leicht zu erkennen sind. $\mathrm{Da}$ die beiden Kephaline Phosphatidylserin und Phosphatidyl-aethanolamin vielfach nicht gleich hoch ansteigen, ist es angezeigt, den für die quantitative Bestimmung auszukratzenden Bereich genügend groß zu wählen.

\section{Statistische Versuche zur Ermittlung der Feblerbreite}

Ein und dasselbe Serum wurde fünfmal nach der unter „Methodik" angegebenen Vorschrift aufgearbeitet, wobei die Bestimmungen je im Abstand von 2 Tagen durchgeführt wurden. In Abänderung der angegebenen Methode wurden jedoch alle 3 Lipoidextrakte je einmal chromatographiert, einer davon auf einer separaten Platte am nächstfolgenden Tag (Resultate s. Tab.6-11).

Tab. 6

Gesamtlipoídbestimmungen

\begin{tabular}{|c|c|c|c|c|}
\hline $\begin{array}{l}\text { Versuch } \\
\text { Nr. }\end{array}$ & $\begin{array}{l}\text { mg Gesamt- } \\
\text { lipoid } / \mathrm{m} l \\
\text { Serum }\end{array}$ & $\begin{array}{l}\text { Mittel- } \\
\text { wert }\end{array}$ & $\begin{array}{l}\text { Standard- } \\
\text { Abweichung }\end{array}$ & $\begin{array}{c}\text { Variations- } \\
\text { koeffizient } \\
\%\end{array}$ \\
\hline $\begin{array}{r}1 \mathrm{a} \\
\mathrm{b} \\
\mathrm{c} \\
2 \mathrm{a} \\
\mathrm{b} \\
\mathrm{c} \\
3 \mathrm{a} \\
\mathrm{b} \\
\mathrm{c} \\
4 \mathrm{a} \\
\mathrm{b} \\
\mathrm{c} \\
5 \mathrm{a} \\
\mathrm{b} \\
\mathrm{c}\end{array}$ & $\begin{array}{l}7,05 \\
6,92 \\
6,90 \\
6,98 \\
6,90 \\
6,98 \\
7,07 \\
7,01 \\
6,93 \\
6,97 \\
6,89 \\
6,98 \\
7,03 \\
7,01 \\
6,91\end{array}$ & $\begin{array}{l}6,95 \\
7,00 \\
6,94 \\
6,98\end{array}$ & $\begin{array}{l}0,0816 \\
0,0463 \\
0,0704 \\
0,0495 \\
0,0644\end{array}$ & $\begin{array}{l} \pm 1,17 \\
\pm 0,67 \\
\pm 1,1 \\
\pm 0,71 \\
\pm 0,92\end{array}$ \\
\hline
\end{tabular}

Tab. 7

Phosphatidbestimmungen

Werte in \% Phosphatide vom Gesamtlipoid Mittelwert aus je 3 Werten

\begin{tabular}{cc}
\hline $\begin{array}{c}\text { Versuch } \\
\text { Nr. }\end{array}$ \\
\hline 1 a & $28, \overline{7}$ \\
b & 28,2 \\
c & 28,3 \\
2 a & 27,9 \\
b & 28,5 \\
c & 28,4 \\
3 a & 28,1 \\
b & 28,8 \\
c & 28,2 \\
4 a & 29,0 \\
b & 28,1 \\
c & 28,6 \\
5 a & 28,5 \\
b & 28,0 \\
c & 28,1 \\
\hline
\end{tabular}


Tab. 8

Bestimmung von Kephalin

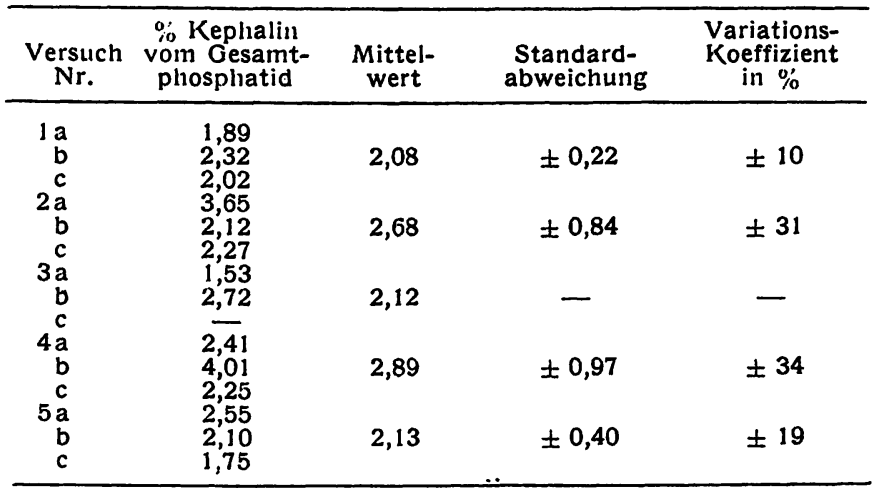

Tab. 9

Bestimmung von Lecithin

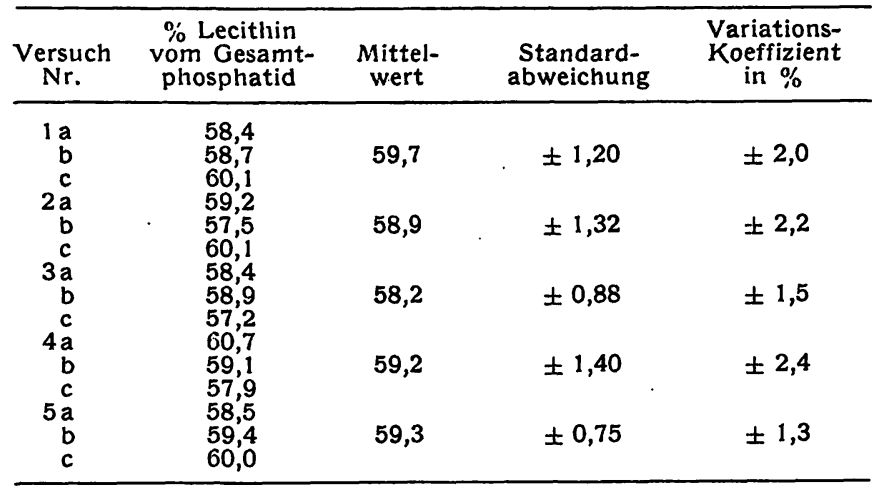

\section{Diskussion}

Unsere vergleichenden Versuche über die Extraktion der Lipoide aus dem Serum bestätigen eine Reihe von Arbeiten $(21,22,26)$, aus denen hervorgeht, daß auch bei starker Reduktion des Extraktionsvolumens die Ausbeute an extrahiertem Lipoid gegenüber der ursprünglichen Folcr-Technik nicht geringer wird. Die Verbesserung der Lipoidextraktion durch die von Folch und Mitarbeitern (19) eingeführten Chloroform-Methanol-Mischungen gegenüber der BLOOR-Variante mit Äthanol-Äther kam aụch bei unserer Versuchsanordnung deutlich zum Ausdruck.

Die dünnschichtchromatographische Auftrennung der extrahierten Serumlipoide bietet heute kaum mehr Schwierigkeiten, bestehen doch eine ganze Reihe von sehr guten Vorschriften (6, 27, 13, 15, 7, 12). Die besten Resultate erhielten wir im Hinblick auf die saubere Trennung der Phosphatide mit der von Jatzkewrtz (11) vorgeschlagenen 2-Stufen-Technik, nämlich ChloroformMethanol- $\mathrm{H}_{2} \mathrm{O}$ (14:6:1) und Propanol-12,5 proz. Ammoniak (4:1).

Bei der Identifizierung und speziell der Abgrenzung der Kephalinfraktion können gewisse Schwierigkeiten auftreten, einerseits wegen der sehr geringen Menge dieses Phosphatides, andererseits wegen der nicht sẹt gut reproduzierbaren $R_{\mathrm{F}}$-Werte des Serin-Kephalins und Aethanolamin-Kephalins. Erschwerend wirkt sich zudem aus, daß eine Sichtbarmachung mit Ninhydrinreagenz nicht durchgeführt werden kann, weil dann die anschließende Extraktion aus dem Kieselgel nicht mehr quantitativ verläuft. Wie weiter unten bei der Diskussion der Genauigkeit unserer Methode noch ausgeführt wird,
Tab. 10

Bestimmung von Sphingomyelin

\begin{tabular}{|c|c|c|c|c|}
\hline $\begin{array}{l}\text { Versuch } \\
\text { Nr. }\end{array}$ & $\begin{array}{l}\% \text { Sphingomyelin } \\
\text { vom Gesamt- } \\
\text { phosphatid }\end{array}$ & $\begin{array}{c}\text { Mittel- } \\
\text { wert }\end{array}$ & $\begin{array}{l}\text { Standard- } \\
\text { abweichung }\end{array}$ & $\begin{array}{l}\text { Variations- } \\
\text { Koeffizient } \\
\text { in \% }\end{array}$ \\
\hline $\begin{array}{r}1 \mathrm{a} \\
\mathrm{b} \\
\mathrm{c} \\
2 \mathrm{a} \\
\mathrm{b} \\
\mathrm{c} \\
3 \mathrm{a} \\
\mathrm{b} \\
\mathrm{c} \\
4 \mathrm{a} \\
\mathrm{b} \\
\mathrm{c} \\
5 \mathrm{a} \\
\mathrm{b} \\
\mathrm{c}\end{array}$ & $\begin{array}{l}16,9 \\
18,2 \\
17,1 \\
17,2 \\
18,0 \\
18,1 \\
17,4 \\
16,8 \\
18,2 \\
18,0 \\
17,5 \\
17,4 \\
16,7 \\
17,3 \\
18,3\end{array}$ & $\begin{array}{l}17,4 \\
17,8 \\
17,5 \\
17,6 \\
17,4\end{array}$ & $\begin{array}{l} \pm 0,70 \\
\pm 0,49 \\
\pm 0,70 \\
\pm 0,32 \\
\pm 0,81\end{array}$ & $\begin{array}{l} \pm 4,0 \\
\pm 2,7 \\
\pm 4,0 \\
\pm 1,8 \\
\pm 4,6\end{array}$ \\
\hline
\end{tabular}

Tab. 11

Bestimmung von Lysolecithin

\begin{tabular}{|c|c|c|c|c|}
\hline $\begin{array}{l}\text { Versuch } \\
\text { Nr. }\end{array}$ & $\begin{array}{l}\% \text { Lysolecithin } \\
\text { vom Gesamt- } \\
\text { phosphatid }\end{array}$ & $\begin{array}{l}\text { Mittel- } \\
\text { wert }\end{array}$ & $\begin{array}{l}\text { Standard- } \\
\text { abweichung }\end{array}$ & $\begin{array}{l}\text { Variations- } \\
\text { Koeffizient } \\
\text { in \% }\end{array}$ \\
\hline $\begin{array}{r}1 \mathrm{a} \\
\mathrm{b} \\
\mathrm{c} \\
2 \mathrm{a} \\
\mathrm{b} \\
\mathrm{c} \\
3 \mathrm{a} \\
\mathrm{b} \\
\mathrm{c} \\
4 \mathrm{a} \\
\mathrm{b} \\
\mathrm{c} \\
5 \mathrm{a} \\
\mathrm{b} \\
\mathrm{c}\end{array}$ & $\begin{array}{r}9,57 \\
8,90 \\
8,55 \\
10,30 \\
8,65 \\
9,05 \\
9,60 \\
9,15 \\
8,35 \\
8,95 \\
10,10 \\
9,30 \\
8,25 \\
9,70 \\
9,95\end{array}$ & $\begin{array}{l}9,45 \\
9,30\end{array}$ & $\begin{array}{l} \pm 0,52 \\
\pm 0,90 \\
\pm 0,63 \\
\pm 0,59 \\
\pm 0,92\end{array}$ & $\begin{array}{l} \pm 5,8 \\
\pm 9,7 \\
\pm 7,0 \\
\pm 6,2 \\
\pm 9,9\end{array}$ \\
\hline
\end{tabular}

ist die Bestimmung des Kephalins mit der hier vorgeschlagenen Technik deshalb nicht sehr befriedigend, und falls die möglichst genaue Bestimmung dieses Phosphatides im Vordergrund steht, müßte eine andere Methode gewählt werden.

Wie die Ausbeutenberechnung (s. Tab. 3) verdeutlicht, findet man nach der Chromatographie nur etwa 85 bis 90\% der aufgetragenen Phosphatide wieder. Die restlichen 10-15\% verteilen sich größtenteils auf die nur in geringen Mengen vorkommenden anderen Phosphatide. Diese finden sich zum Tcil nicht identifizierbar außerhalb, zum Teil aber auch innerhalb der ausgekratzten Zonen (Cardiolipin, Phosphatidsäure, InositPhosphatide, Lysokephalin, Plasmalogene). Dieser Umstand hat eine geringfügige Verfälschung der mit unserer Methode für Kephalin, Lecithin, Sphingomyelin und Lysolecithin bestimmten Werte zur Folge, doch dürfte dieser Fehler, ausgenommen beim Kephalin, kaum merklich ins Gewicht fallen. Geringe Mengen Phosphatid gehen zudem bei der Extraktion aus dem Kieselgel verloren (s. Tab. 5). Die Extraktion der Phosphatide aus dem Kieselgel für die P-Bestimmung ist unseres Erachtens trotz der etwas komplizierteren Technik vorteilhafter als die direkte Veraschung zusammen mit dem Trägermaterial. Die starke Streuung der Blindwerte für Kieselgel allein bringt einen zusätzlichen Fehler und die von Zieve und Mitarbeitern (15) und von Phillips (12) sowie von Christian (8) angegebenen P-Bestimmungs-Techniken ergaben in unseren Versuchen durchweg zu niedrige Werte. PhrrLrps weist in seiner Arbeit auf den inhibierenden Effekt des Kieselgels bei der Farbbildung hin; er korrigiert denn auch alle gefundenen 
P-IWerte um 20\% nach oben, dies basierend auf Experimente, in denen der Inhibitoreffekt verschieden großer Mengen Kieselgel bestimmt und, ausgedrückt als Farbverminderung, zu 11\% - 27\% gefunden wurde.

Die aufgrund der statistischen Berechnungen gefundene Genauigkeit der Methode darf für die Werte der Gesamtlipoide, der Phosphatide, des Lecithins und Sphingomyelins als befriedigend bezeichnet werden. Beim Lysolecithin mit einem Variationskoeffizienten von $\pm 10 \%$ wäre eine größete Genauigkeit sehr wünschenswert, hauptsächlich im Hinblick auf seine eventuelle Wichtig- keit bei der Komplement-Lyse. Sehr fragwürdig bleibt wie bereits erwähnt die Bestimmung des Kephalins (Variationskoeffizient $\pm 34 \%$ ). Die Fehlerbreite bei der von uns vorgeschlagenen Methode ist allerdings immer noch wesentlich geringer als die Variabilität der Lipoidzusammensetzung bei normalen Sera. Tatsächlich scheint heute das. Problem der Feststellung signifikanter $\mathrm{Ab}$ weichungen vom Norm-Wert weit eher durch statistisch einwandfreie Festlegung der Normalverteilung der Gesamtlipoide, als durch die Genauigkeit der analytischen Prozeduren bestimmt zu sein.

\title{
Literatur
}

1. Kaufmann, H. P., Fette und Seifen 46, 268 (1939). - 2. Wren, J. J., J. Chromatogr. 4, 173-195 (1960). - 3. Marinetrr, G. V., J. Lipid Res. 3, 1 (1962). - 4. Wercker, H., Klin. Wochenschr. Jg. 37, Heft 14, 763 (1959). - 5. Mangold, H. K., Fette, Seifen und Anstrichmittel 61, 877 (1959). - 6. WAGNER, H., L. HöRHAMMER und P. WolfF, Biochem. Zeitschr. 334, 175-184 (1961). - 7. Blank, M. L., J. A. Schmit und O. S. Privett, J. Am. Oilchemists Soc. 41, Nr. 5, 371 (1964). - 8. Christian, J. C., S. Jakovic und D. Yr-Yung Hsia, J. Lab. Clin. Med. 5,5 (1964). - 9. Doizakr, W. M. und L. Zieve, Proc. Exp. Biol. Med. 113, 91 (1963). - 10. HabermanN, E. G., G. Bandflow und B. KRusche, Klin. Wschr. 39, 816 (1961). - 11. JatzKewITz, H., a) Hoppe Seyler's Zeitschr. physiol. Chemie 326, 61 (1961), b) Hoppe Seyler's Zeitschr. physiol. Chemie 336, 25 (1964). 12. Robinson, N. und B. M. Phillips, Clin. Chim. Acta 8, 385 (1963). - 13. Skipski, V. P., R. F. Peterson, J. Sanders und M. Barclay, J. Lipid Res. 4, No. 2, 227 (1963). - 14. Vrkrot, O.,
Acta Med. Scand. 175, Fasc. 4, 443 (1964). - 15. VoGEL, W. C. W. M. DoizAkI und L. ZIEVE, J. Lipid Res. 3, 138 (1962). 16. Zöllner, N. und G. Wolfram, Klin. Wochenschr. Jg. 40, Heft 21, 1101 (1962). - 17. Frsk, C. H. und Y. Subba-Row, J. Biol. Chem. 66, 375 (1925). - 18. De Iongh, van PeLt, J. G., J. Lipid Res. 3, No. 3, 385 (1962). - 19. Folch, J., M. LeEs und G. H. Sloane Stanley, J. Biol. Chem. 226, 497 (1957). 20. Bloor, W. R., J. Biol. Chem. 77, 53 (1928). - 21. RENKonen, O., T. U. Kosunen und O. V. Renkonen, Ann. Med. Exp. Biol. Fannia 41, 375 (1963). - 22. Bligh, E. G. und W. J. Dyer, Can. J. Biochem. Physiol. 37, 8 (1959). - 23. Jestrng, E. und H. O. BANG, Scand. J. Clin. Lab. Invest. 15, 654 (1963). - 24. BARTLETT, G. R., J. Biol. Chem. 234, 466 (1959). - 25. Shrn, Y. S., Anal. Chem. 34, 1164 (1962). - 26. Hanson, S. W. F. und J. Olley, Biochem. J. 89, 101 p (1963). - 27. Privetr, O. S., M. L. Blank, D. W. Codding und E. C. Nrckell, J. Am. Oil Chemist Soc. 42, Nr. 5, 381 (1965).

\section{Untersuchungen zur enzymatischen Bestimmung der Galaktose mit Galaktoseoxydase}

\author{
Von H. Förster und M. HASLbeck
}

Aus dem Institut für vegetative Pbysiologie der Universität Frankfurt (Direktor: Prof. Dr. med. E. Heinz) und der III. Medizinischen Klinik des Krankenbauses München-Schivabing (Chefarzt: Priv. Doz. Dr. med. H. Mebnert)

(Eingegangen am 3. Januar 1967)

Die quantitative Galaktosebestimmung mittels Galaktoseoxydase-Peroxydase wurde untersucht. Dabei wurde als optimales Puffersystem Barbitalnatrium-Natriumacetat-Puffer gefunden. - Als Wasserstoffdonator wirkte o-Tolidin am günstigsten. Bestimmungen im Serum und besonders im Harn werden durch Harnsäure gestört. Dabei dienen Harnsäure und auch Glutathion als Wasserstoffdonatoren für die Peroxydasereaktion. Diese beiden Substanzen lassen sich jedoch auf einfache Weise durch Zusatz von Quecksilberacetat zu der als Enteiweißungsmittel dienenden Perchlorsäure eliminieren. Ein weiterer Vorteil des Quecksilberacetatzusatzes ist die dadurch bewirkte Farbvertiefung des Chromogens.

The quantitative determination of galactose by means of the coupled galactose-oxidase peroxidase system was investigated. Sodium barbiturate-sodium acetate buffer was found to be the most suitable system for this work. O-tolidine was the most satisfactory hydrogendonor. Measurements in serum and particularly in urine were disturbed by the presence of uric acid. This is because uric acid and also glutathione may act as hydrogen donors for the peroxidase reaction. These two substances may be simply eliminated by the addition of mercuric acetate to the perchloric acid used as a protein precipitant. A further advantage of mercuric acetate is that it intensifies the colour of the chromogen.

Die Bestimmung der Galaktose im Blut war bisher immer schwierig, da eine spezifische Methode fehlte. Die im Blut vorhandene Glucose stört bei jedem chemischen Nachweis, der auf dem Reduktionsprinzip oder auf dem Furfurolprinzip beruht (1); denn die Blutglucosekonzentration ist meist wesentlich höher als die Blutgalaktosekonzentration, während die chemischen Nachweismethoden für beide Zucker etwa gleich empfindlich sind. Die Reduktionsmethoden exfassen zudem noch andere reduzierende Substanzen als sog. Restreduktion, wäh- rend die o-Toluidin-Methode relativ spezifisch für Hexosen ist. Eine gewisse Verbesserung brachte die von Watson (2) angegebene Methode. Glucose wird durch Glucoseoxydase zerstört und die von diesem Ferment nicht angegriffene Galaktose wird anschließend mit oToluidin bestimmt. $\mathrm{Da}$ aber die Glucoseoxydation mit Glucoseoxydase meist unvollständig ist, sind die Fehlermöglichkeiten dieser Methode immer noch groß (3). Neue Möglichkeiten ergaben sich aus der Isolierung der Galaktoseoxydase aus Dactylium dendroides (4-8). Die 